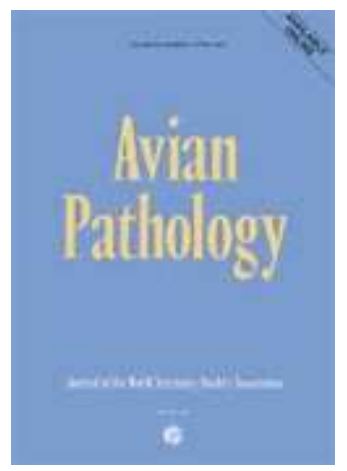

\title{
OUTBREAK OF DISEASE ASSOCIATED WITH CRYPTOSPORIDIA ON A RED-LEGGED PARTRIDGE (ALECTORIS RUFA) GAME FARM
}

\begin{tabular}{|c|c|}
\hline Journal: & Avian Pathology \\
\hline Manuscript ID: & CAVP-2006-0150.R1 \\
\hline Manuscript Type: & Original Research Paper \\
\hline $\begin{array}{r}\text { Date Submitted by the } \\
\text { Author: }\end{array}$ & 09-Mar-2007 \\
\hline Complete List of Authors: & $\begin{array}{l}\text { Pagès-Manté, Albert; Laboratorios Hipra S.A. } \\
\text { Pagès, Marc; Laboratorios Hipra S.A. } \\
\text { Majó-Masferrer, Natalia; Universidad Autónoma de Barcelona, } \\
\text { Animal health and Anatomy } \\
\text { Gómez-Couso, Hipólito; University of Santiago de Compostela, } \\
\text { Microbiology and Parasitology } \\
\text { Ares-Mazás, Elvira; University of Santiago de Compostela, } \\
\text { Microbiology and Parasitology }\end{array}$ \\
\hline Keywords: & $\begin{array}{l}\text { Red-legged partridge, Cryptosporidium meleagridis, histological, } \\
\text { immunological and molecular diagnosis, Outbreak of disease }\end{array}$ \\
\hline
\end{tabular}

\section{SCHOLARONE" \\ Manuscripts}




\title{
An outbreak of disease associated with cryptosporida on a red-legged partridge (Alectoris rufa) game farm
}

\author{
A. Pagès-Manté ${ }^{1}$, M. Pagès-Bosch ${ }^{1}$, N. Majó-Masferrer ${ }^{2}$, H. Gómez-Couso ${ }^{3}$, E. \\ Ares-Mazás ${ }^{3}$
}

${ }^{1}$ Laboratorios Hipra S.A., Avda. La Selva, 135. 17170 Amer, Girona, Spain

${ }^{2}$ Departamento de Sanidad y Anatomía Animal, Facultad de Veterinaria, Campus de la Universidad Autónoma de Barcelona. 08193 Bellaterra (Cerdanyola del Vallès),

Barcelona, Spain

${ }^{3}$ Laboratorio de Parasitología, Departamento de Microbiología y Parasitología, Facultad de Farmacia, Universidad de Santiago de Compostela. 15782 Santiago de Compostela, A Coruña, Spain

Running Head: Cryptosporidia in red-legged partridge

*To whom correspondence should be addressed. Tel.: +34 981563 100; fax: +34 981 593 316. E-mail address: mpeares@usc.es

Received: 4 October 2006 
Cavp-2006-0150

\title{
An outbreak of disease associated with cryptosporida on a red-legged partridge (Alectoris rufa) game farm
}

\author{
A. Pagès-Manté ${ }^{1}$, M. Pagès-Bosch ${ }^{1}$, N. Majó-Masferrer ${ }^{2}$, H. Gómez-Couso ${ }^{3}$, E. $^{-}$ \\ Ares-Mazás ${ }^{3 *}$
}

Abstract

An outbreak of disease associated with cryptosporidia on a red-legged partridge (Alectoris rufa) game farm is described. Morbidity (diarrhoea and cough) was between 60 and $70 \%$ during the first weeks of life (4 to 25 days) and mortality was higher than $50 \%$. The results of bacteriological and virological analyses were negative. Histological examination and antigenic diagnosis by ELISA revealed the presence of Cryptosporidium spp. in respiratory and intestinal tracts. The application of PCR-RFLP techniques and sequencing of a COWP gene fragment confirmed the existence of C. meleagridis in faecal samples. The results obtained suggest that avian cryptosporidiosis should be included among respiratory and enteric diseases routinely tested for in farmed birds. 


\section{Introduction}

Species belonging to the genus Cryptosporidium are monoxenous protozoan parasites that infect a wide range of vertebrate hosts, including humans (Fayer, 2004). Cryptosporidium spp. cause acute, self-limiting gastroenteritis in immunocompetent individuals, and chronic and potentially fatal infections in immunocompromised subjects worldwide (Cacciò et al., 2005). In animals, cryptosporidiosis appears mainly to be a problem in neonatal ruminants, and causes considerable direct and indirect economic losses (de Graaf et al., 1999).

Avian cryptosporidiosis is an emerging health problem in the poultry industry and is associated with respiratory/intestinal disease that can cause severe morbidity and mortality (de Graaf et al., 1999). The birds can become infected by ingestion or inhalation and/or inspiration of oocysts present in the environment (litter, faeces, water, nesting materials, dust, etc.). As few as 100 oocysts can cause intestinal or respiratory infections (Sréter \& Varga, 2000) and because of the limited availability of effective drugs, hygienic conditions and management strongly influence the incidence and persistence of avian cryptosporidiosis (Current, 1997; de Graaf et al., 1999).

Although infections have been found in over 30 host species, including domestic, caged and wild birds, only three species of Cryptosporidium affecting avian hosts have been named on the basis of their morphological, biological and molecular differences (Xiao et al., 2004). Cryptosporidium meleagridis (Slavin, 1955) may infect the intestinal tract, bursa of Fabricious (BF) and cloaca of turkeys and chickens, but infection has previously only been associated with illness, including diarrhoea and moderate mortality in turkeys. Oocysts measure $5.2 \times 4.6 \mu \mathrm{m}$ (Sréter \& Varga, 2000). 
Cryptosporidium baileyi (Current et al., 1986) may infect the respiratory tract (larynx, trachea, primary and secondary bronchi and air sacs), BF and cloaca mainly of chickens, turkeys and ducks. It has also been described in quails (Morgan et al., 2001). C. baileyi is the most prevalent species of Cryptosporidium in poultry and the most commonly associated with disease in chickens. Oocysts of $C$. baileyi measure $6.2 \times 4.6$ $\mu \mathrm{m}$ (Sréter \& Varga, 2000).

A third species of avian Cryptosporidium was first reported by Pavlasek (1999, 2001) and recently re-described as Cryptosporidium galli (Ryan et al., 2003). The oocysts are ellipsoidal (size range 8.0-8.0 ×6.2-6.4 $\mu \mathrm{m}$ ) and the life cycle stages develop in the epithelial cells of the proventriculus in a variety of birds. Moreover, new genotypes of avian cryptosporidia have been found in different hosts, and may represent separate species (Xiao et al., 2004).

In the present study, an outbreak of disease associated with cryptosporidia on a game farm of red-legged partridge (Alectoris rufa) is described.

\section{Materials and Methods}

Case history. The case occurred on a red-legged partridge game farm in Cataluña (northeast Spain) on which there were 100 breeding pairs. An outbreak of disease, characterized by diarrhoea and coughing, was detected in May 1999, coinciding with the first hatches of the season. From 4 to 5 days of age, the chicks produced a higherthan-usual number of depositions/caecal discharges of a pasty consistency, and on some occasions a watery halo was observed in normal faeces. In addition, approximately $10 \%$ of the animals had a dry cough and sometimes a nasal discharge. A few days after the 
appearance of the clinical signs, the birds became apathetic, and then prostrate, with dropping wings, trembling and, following slight paralysis, they died in a lateral prone position. Morbidity varied between 60 to $70 \%$ between 4 and 25 days of age, with a mortality rate of $50 \%$. From of 30 days of age onwards, the surviving birds developed and behaved as normal after removal of between 2 to $3 \%$ of birds that remained affected. The situation occurred again with later hatches until July, causing considerable economic loss on the farm. In July the condition became more severe as the condition lasted for more than 30 days and caused greater mortality than before. Of the last 450 chicks that hatched, only 50 survived ( $89 \%$ mortality). During the outbreak (May to July), the birds were treated therapeutically and prophylactically in the drinking water with several drugs: ipronidazol (200 mg/l, for 5 days), sulfaquinoxaline (75 mg/l, two periods of three days separated by two rest days) and enrofloxacin (100 mg/l for 5 days). Additional biosecurity measures were introduced to physically separate the different hatches to avoid cross-contamination. Huts used for the youngest birds were thoroughly cleaned and disinfected using water with formaldehyde and salt. Daily movement of personnel was also reviewed in order to avoid cross-contamination. In addition, changes of shoes and clothing between huts were implemented. Special precautions were introduced to prevent access of wild birds (mainly sparrows) and rodents to the facilities by using appropriate nets and authorized traps.

Histopathology. Four affected partridges (two 11 days-old and two 25 days-old) were anaesthetized with thiopental sodium and killed by exsanguination. At autopsy, trachea, bronchi, lungs and intestinal organs (duodenum and caecum) were removed for bacteriological, viral and parasitological analyses, and the remaining parts of these organs were fixed in $10 \%$ neutral buffered formalin for histopathological examination. 
After fixation, samples were embedded in paraffin, sectioned at 3 to $4 \mu \mathrm{m}$ and stained with haematoxylin-eosin following routine histological methods.

Bacteriology. Samples of trachea, lung, duodenum and caecum were inoculated directly into TSA medium, modified brilliant green medium and Columbia III w/5\% SB medium (ref. 254051, 254490 \& 254097 respectively; BD Diagnostic, Diagnostic Systems, Sparks, MD, USA) and incubated at $37^{\circ} \mathrm{C}$ for $72 \mathrm{~h}$. For isolation of Mycoplasma spp., samples of trachea and lung were initially inoculated into Mycoplasma broth base (PPLO, BD Diagnostics, Diagnostic Systems) supplemented with phenol red, glucose, horse serum, NAD, cysteine, thallium acetate and penicillin according to Frey et al. (1968) and incubated at $37^{\circ} \mathrm{C}$ in a atmosphere containing $10 \%$ $\mathrm{CO}_{2}$ (BB-16, Heraeus, Munchen, Germany) for 7 days. Samples from the broth culture were then streaked on to Mycoplasma agar prepared as above but with $1.3 \%$ agar (ref. 214230; BD Diagnostics, Diagnostic Systems), maintained under identical conditions, and examined daily for 25 days.

Virology. For virus isolation, 9-days-old embryonated specified pathogen free (SPF) chicken eggs (Lohmann Gmb H, Cuxhaven, Germany) and primary fibroblast monolayer cultures were used according to Rovozzo \& Burke (1973). Lung and intestine samples (duodenum and caecum) were suspended at a ratio of 1:10 (w/v) in phosphate buffered saline (PBS, pH 7.2) and mechanically homogenized (Stomacher ${ }^{\circledR}$ 400, Seward, Worthing, UK). The samples were then centrifuged at $3000 \times g$ for $5 \mathrm{~min}$. The supernatants were filtered through nitrocellulose filters $(2 \mu \mathrm{m}$ diameter pore $)$ (Millipore Corporation, Bedford, USA) and $0.2 \mathrm{ml}$ aliquots of filtrate were inoculated into each of three SPF eggs (via the allantoic cavity) and two Falcon ${ }^{\mathrm{TM}}$ cell culture tubes 
(BD Biosciences, San Jose, CA, USA) containing fibroblast monolayers, which were maintained for 4 days at $37^{\circ} \mathrm{C}$.

Four days post inoculation, the eggs were opened aseptically and compared with the controls to detect any anomaly that indicated presence of a virus. Ten ml aliquots of allantoic fluid were collected and passaged twice following the same methodology. Likewise, at 4 days post inoculation, the fibroblast monolayers were examined microscopically $(10 \times)$ to check for cytopathic effects or differences in development compared with control cultures. Two further passages were carried out with $5 \mathrm{ml}$ aliquots of the fibroblast culture supernatants. In addition, the faeces were examined for rotavirus type A by an ELISA capture test, in accordance with the manufacturer's instructions (Ingezim Rotavirus Das 1.1.RT.K.2, Ingenasa, Madrid, Spain).

Parasitology. Faecal samples from the four necropsied birds were processed by a saturated sugar (density $1.27 \mathrm{~g} / \mathrm{ml}$ ) flotation technique and pools of homogenized samples from trachea, bronchi, lung, duodenum, caecum and faeces were used for antigenic diagnosis of Cryptosporidium by ELISA (Bio-X Cryptosporidium parvum ELISA kit, Bio-X diagnostics, Jemelle, Belgium), according to the manufacturer's instructions.

PCR-RFLP and sequencing analyses for Cryptosporidium spp. A modification of Boom's method (Boom et al., 1990), as previously described by McLauchlin et al. (1999), was used to extract nucleic acids from faecal samples. Briefly, $200 \mu 1$ of the sample were homogenized with zirconia beads using a Mini-Bead-Beater-8 ${ }^{\mathrm{TM}}$ (BioSpec Products, Inc., Batlesville, Oklahoma, USA) in guanidinium thiocyanate solutions. Nucleic acids released from disrupted oocysts were purified with coarse activated silica 
suspensions (Severn Biotech, Ltd., Kidderminster, UK), and stored at $-20^{\circ} \mathrm{C}$ until use. A fragment of $341 \mathrm{bp}$ from the Cryptosporidium oocyst wall protein (COWP) gene was amplified by a nested PCR procedure, with the PCOWPF/PCOWPR and PCOWPIF/PCOWPIR primers in the first and second phase respectively, as described elsewhere (Gómez-Couso et al., 2004). DNA extracted from isolates of Cryptosporidium parvum and C. meleagridis, and water instead of DNA template were used as positive and negative controls, respectively. PCR products were visualized using $2 \%$ agarose gels stained with ethidium bromide. Restriction fragment length polymorphism (RFLP) analyses were carried out with $R s a \mathrm{I}$ and $A l u \mathrm{I}$ enzymes (Stratagene, La Jolla, California, USA). The RFLP analyses were performed by digesting $10 \mu \mathrm{l}$ of PCR product with $5 \mathrm{U}$ of each restriction enzyme in $1 \times$ enzyme buffer (Stratagene), in a final volume of $30 \mu \mathrm{l}$, for $4 \mathrm{~h}$ at $37^{\circ} \mathrm{C}$ (Gómez-Couso et al., 2004). Restriction fragments were analysed by electrophoresis in $3.2 \%$ agarose gels stained with ethidium bromide. PCR products were purified with Quantum Prep ${ }^{\circledR}$ PCR Kleen Spin Columns (Bio-Rad Laboratories, Hercules, California, USA) and sequenced with the CEQ ${ }^{\mathrm{TM}}$ DTCS- Quick Start Kit (Beckman Coulter, Inc., Fullerton, California, USA) in both directions, with inner primers of the nested PCR. Sequencing reactions were analysed with a Beckman Coulter CEQ ${ }^{\text {TM }} 8000$ Genetic Analysis System (Beckman Coulter, Inc.). Sequence data was examined by the BioEdit Sequence Alignment Editor v7.0.1 (Copyright $(\subset)$ 1997-2004 Tom Hall, Ibis Therapeutics, Carlsbad, California, USA), and Chromas v2.3 (Copyright $(1)$ 1998-2004 Technelysium Pty Ltd, Tewantin Qld, Australia) programmes. The resulting sequences were compared with those of Cryptosporidium spp. deposited in the GenBank. 


\section{Results}

The therapeutic and prophylactic administration of different drugs provided only slight improvement of clinical signs, and did not prevent the appearance of the disease in subsequent hatches, or reduce the rate of mortality.

Autopsy of dead 11-day-old partridges revealed macroscopic thickening of the small and large intestine walls and inflammation and brownish-yellow contents of the caecum. Similar intestinal contents were observed in the 25 -day-old chicks, in addition to slight congestion of the lungs, although the other organs were of normal appearance.

Microscopic examination revealed numerous small, round parasitic structures, 4 to $6 \mu \mathrm{m}$ in diameter, suggestive of Cryptosporidium spp., in the epithelial brush border of respiratory and intestinal samples from the four autopsied partridges (Figure 1).

Occasionally epithelial hyperplasia with moderate heterophilic infiltration was also seen.

The bacteriological studies showed the presence only of Escherichia coli at intestinal level, which was possibly unrelated to the case given its sensitivity to the antibiotics tested. No Mycoplasma organisms were isolated nor were any viruses isolated in SPF eggs or fibroblast monolayers, after three consecutive blind passages. The tests for rotavirus type A by the ELISA capture technique were also negative.

Faecal samples analysed by the saturated sugar flotation technique did not reveal parasitic forms but the ELISA for the antigenic diagnosis of Cryptosporidium gave positive results in all the pools analysed (net optical densities $>0.150$ ). Finally, the application of PCR-RFLP techniques (Figure 2) and sequencing of a COWP gene fragment confirmed the existence of $C$. meleagridis in faecal samples, with a sequence showing $100 \%$ similarity to the AF266266 sequence deposited in the GenBank. 
Biosecurity procedures introduced before the next reproductive season gave good results and no other problems were recorded on this farm.

\section{Discussion}

For many years avian cryptosporidiosis has been considered to be an opportunistic disease. However, new data indicate that this parasitic disease is a serious problem in the poultry industry (de Graaf et al., 1999). In chickens and other Galliformes, cryptosporidiosis is mostly manifested as a respiratory disease, although enteric disease has also been reported in turkeys and quails. Cryptosporidiosis is highly prevalent in chicken flocks (Sréter \& Varga, 2000), and its pathogenicity increases when the infection coexists with immunosuppressive or respiratory agents, even when birds are vaccinated against other pathogens (de Graaf et al., 1999).

The present report is the first description of an outbreak of disease associated with $C$. meleagridis in red-legged partridge reared on a game farm. The association was confirmed by the application of PCR-RFLP techniques and the sequencing of a COWP gene fragment from faecal samples, which provided a nucleotide sequence showing $100 \%$ similarity to C. meleagridis. The findings of the present study also extend the range of hosts of $C$. meleagridis, the potential aetiological agent responsible for high mortality during the first weeks of life of these birds. The pathogen may have contributed to the repeated disappearance of partridge flocks during their natural breeding period, as reported by rangers and gamekeepers, who have detected high mortality among recently hatched birds.

The observation of Cryptosporidium-like bodies in histological sections of the respiratory tract, in addition to the positive results obtained by ELISA with pools of 
homogenized samples from trachea, bronchi and lung and the clinical symptoms of cough and nasal discharge observed in a small number of birds suggest a co-infection with $C$. baileyi. However, it could be possible that $C$. meleagridis spreads to the respiratory tract as may happen with $C$. parvum during the course of severe infections (Mascaró et al., 1994; Hunter \& Nichols, 2002).

Avian cryptosporidiosis can be spread via several different routes of transmission. Drinking water may serve as a primary route of distribution of oocysts among poultry flocks. Since avian Cryptosporidium species can infect a wide variety of birds, wild birds may play an important role in the epidemiology of cryptosporidiosis. Moreover, since some rodents also appear to be susceptible to $C$. meleagridis under experimental conditions, it is thus possible that mice, voles, and rats could also serve as carriers (see Sréter \& Varga, 2000).

It is also important to consider that $C$. meleagridis may infect and cause cryptosporidiosis in farm personnel as shown with $C$. parvum and C. hominis (McLauchlin et al., 2000). Moreover, infection with C. meleagridis may have a similar prevalence in humans to that of C. parvum, as found in Peru (Cama et al., 2003).

In view of the detection of cryptosporidia in abnormal organs at necropsy and the detection by PCR-RFLP and sequence analyses of $C$. meleagridis in faecal samples, we believe that Cryptosporidium spp. could account for this outbreak of disease, although we cannot completely exclude the possibility other pathogens/non-infectious causes of morbidity and mortality. To do this it would be necessary to examine a greater number of samples and to carry out more extensive tests to rule out other possible causes. For example, electron microscopy on faecal samples for rotaviruses and other enteric viruses would have been helpful, especially since the ELISA used here only detects group A rotaviruses. 
In view of the important economic losses associated with avian cryptosporidiosis and the findings associated with the outbreak described herein, we consider that tests for cryptosporidia should be included when investigating respiratory and enteric disease in farmed birds.

\section{References}

Boom, R., Sol, C.J., Salimans, M.M., Jansen, C.L., Wertheim-van Dillen, P.M. \& van der Noordaa, J. (1990). Rapid and simple method for purification of nucleic acids. Journal of Clinical Microbiology, 28, 495-503.

Cacciò, S.M., Thompson, R.C., McLauchlin, J. \& Smith, H.V. (2005). Unravelling Cryptosporidium and Giardia epidemiology. Trends in Parasitology, 21, 430-437.

Cama, V.A., Bern, C., Sulaiman, I.M., Gilman, R.H., Ticona, E., Vivar, A., Kawai, V., Vargas, D., Zhou, L. \& Xiao, L. (2003). Cryptosporidium species and genotypes in HIV-positive patients in Lima, Peru. Journal of Eukaryotic Microbiology, 50, S531-S533.

Current, W.L. (1997). Cryptosporidiosis. In B.W. Calnek, H.J. Barnes, C.W. Beard, L.R. McDougald \& Y.M. Saif. (1997). Diseases of Poultry 10th edn (pp. 883-890). Ames: Iowa State University Press.

Current, W.L., Upton, S.J. \& Haynes, T.B. (1986). The life cycle of Cryptosporidium baileyi n. sp. (Apicomplexa, Cryptosporidiidae) infecting chickens. Journal of Protozoology, 33, 289-296.

de Graaf, D.C., Vanopdenbosch, E., Ortega-Mora, L.M., Abbassi, H. \& Peeters, J.E. (1999). A review of the importance of cryptosporidiosis in farm animals. International Journal for Parasitology, 29, 1269-1287. 
Fayer, R. (2004). Cryptosporidium: a water-borne zoonotic parasite. Veterinary Parasitology, 126, 37-56.

Frey, M.L., Hanson, R.P. \& Anderson, D.P. (1968). A medium for the isolation of avian mycoplasmas. American Journal of Veterinary Research, 29, 2163-2171.

Gómez-Couso, H., Freire-Santos, F., Amar, C.F., Grant, K.A., Williamson, K., AresMazás, M.E. \& McLauchlin, J. (2004). Detection of Cryptosporidium and Giardia in molluscan shellfish by multiplexed nested-PCR. International Journal of Food Microbiology, 91, 279-288.

Hunter, P.R. \& Nichols, G. (2002) Epidemiology and clinical features of Cryptosporidium infection in immunocompromised patients. Clinical Microbiology Reviews, 15, 145-154.

Mascaró, C., Arnedo, T. \& Rosales, M.J. (1994). Respiratory cryptosporidiosis in a bovine. Journal of Parasitology, 80, 334-336.

McLauchlin, J., Pedraza-Díaz, S., Amar-Hoetzeneder, C. \& Nichols, G.L. (1999). Genetic characterization of Cryptosporidium strains from 218 patients with diarrhea diagnosed as having sporadic cryptosporidiosis. Journal of Clinical Microbiology, 37, 3153-3158.

McLauchlin, J., Amar, C., Pedraza-Díaz, S. \& Nichols, G.L. (2000). Molecular epidemiological analysis of Cryptosporidium spp. in the United Kingdom: results of genotyping Cryptosporidium spp. in 1,705 fecal samples from humans and 105 fecal samples from livestock animals. Journal of Clinical Microbiology, 38, 39843990.

Morgan, U.M., Monis, P.T., Xiao, L., Limor, J., Sulaiman, I., Raidal, S., O’Donoghue, P., Gasser, R., Murray, A., Fayer, R., Blagburn, B.L., Lal, A.A. \& Thompson, 
R.C.A. (2001). Molecular and phylogenetic characterisation of Cryptosporidium from birds. International Journal for Parasitology, 31, 289-296.

Pavlasek, I. (1999). Cryptosporidia: Biology, diagnosis, host spectrum, specificity and the environment. Remedia-Klinicka Mikrobiologie, 3, 290-301.

Pavlasek, I. (2001). Findings of Cryptosporidia in the stomach of chickens and of exotic and wild birds. Veterinarstvi, 51, 103-108.

Rovozzo, G.C. \& Burke, C.N.A. (1973). Manual of Basic Virological Technics. Englewood Cliffs: Prentice Hall Editor.

Ryan, U.M., Xiao, L., Read, C., Sulaiman, I.M., Monis, P., Lal, A.A., Fayer, R. \& Pavlasek, I. (2003). A redescription of Cryptosporidium galli Pavlasek, 1999 (Apicomplexa: Cryptosporidiidae) from birds. Journal of Parasitology, 89, 809813.

Slavin, D. (1955). Cryptosporidium meleagridis (sp. nov.). Journal of Comparative Pathology, 65, 262-266.

Srèter, T. \& Varga, I. (2000). Cryptosporidiosis in birds--a review. Veterinary Parasitology, 87, 261-279.

Xiao, L., Fayer, R., Ryan, U. \& Upton, S.J. (2004). Cryptosporidium taxonomy: recent advances and implications for public health. Clinical Microbiology Reviews, 17, 72-97. 


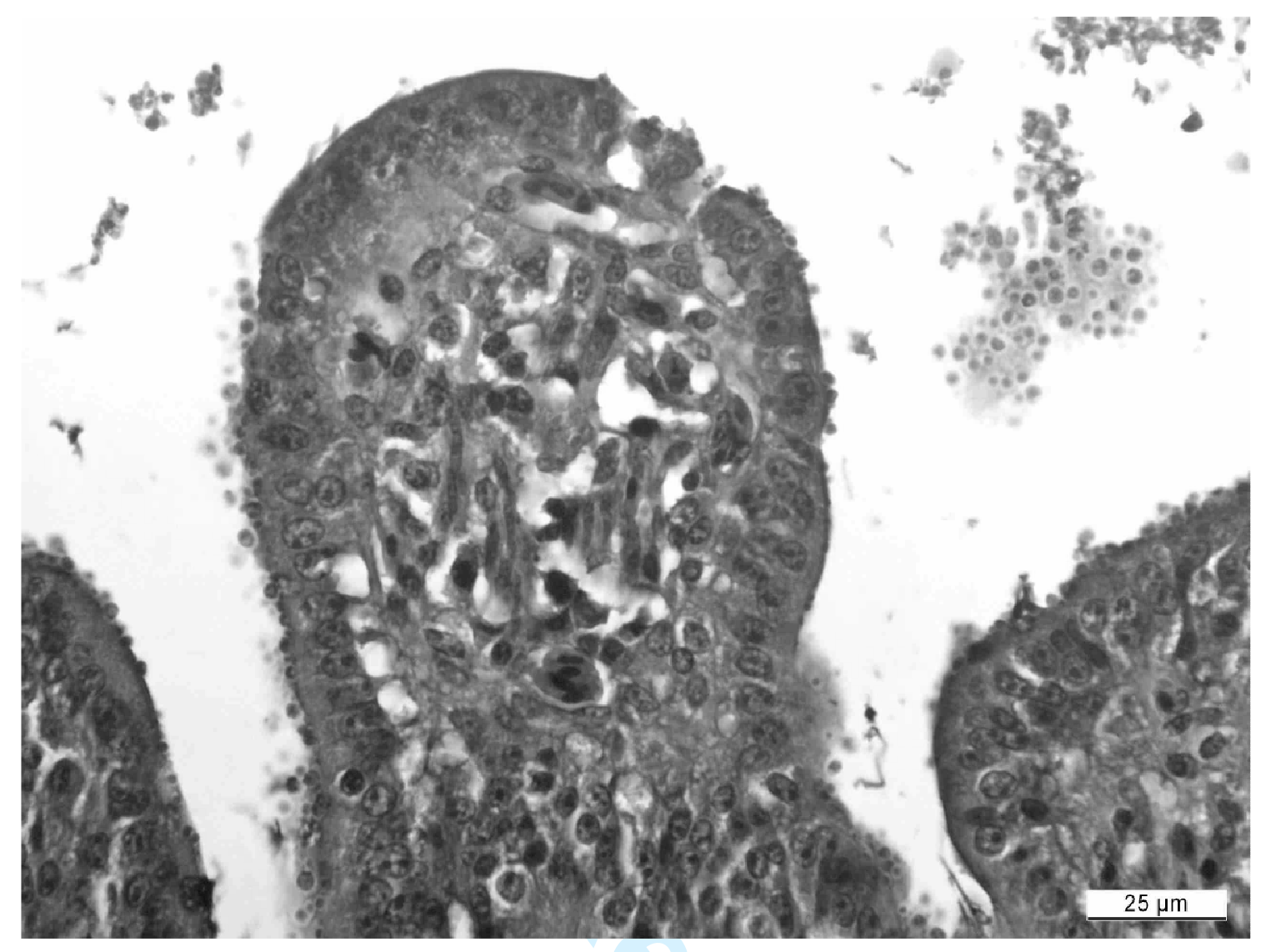

Figure 1. Intestinal section of red partridge (Alectoris rufa) stained with haematoxylineosin showing development stages of Cryptosporidium sp. in the brush border. 


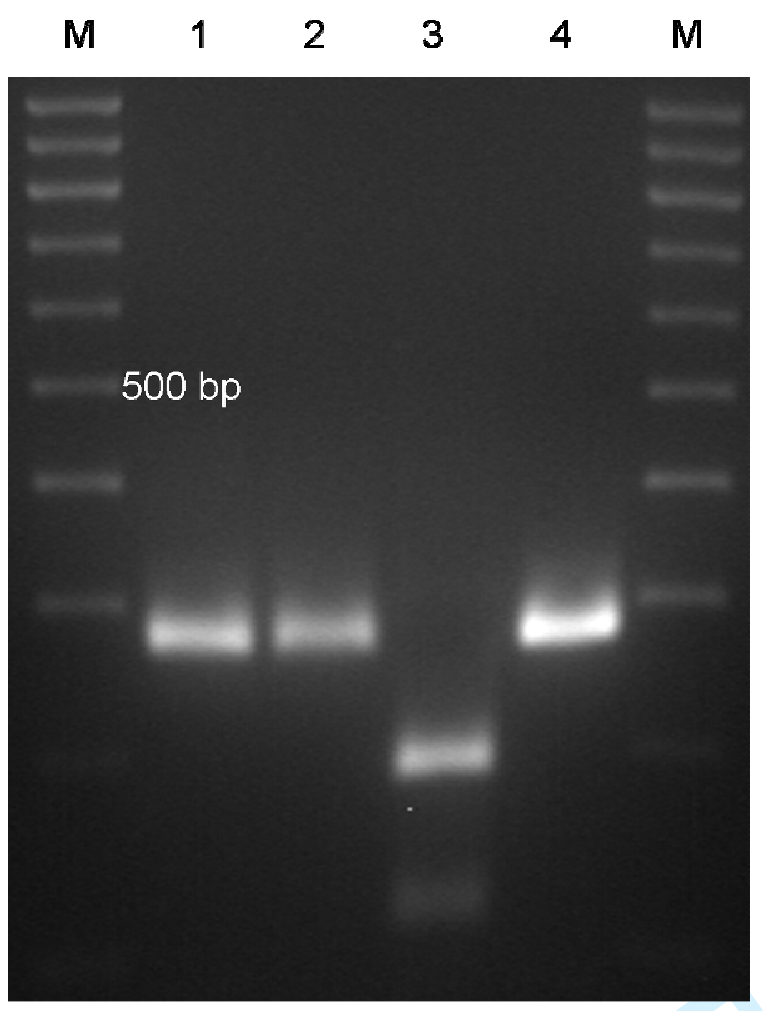

Figure 2. PCR-RFLP of a 341 bp fragment of the Cryptosporidium oocyst wall protein gene using both AluI and RsaI restriction enzymes. Lanes M, 100 bp molecular ladders; lanes 1 and 2, Cryptosporidium isolates from red partridge (Alectoris rufa); lane 3, C. parvum control; lane 4, C. meleagridis control. 